\title{
Atrator de trajetórias de uma equação hiperbólica
}

\author{
Luiz Augusto F. de Oliveira, \\ Depto de Matemática, IME, USP, \\ 05508-090, São Paulo, SP \\ E-mail: luizaug@ime.usp.br, \\ Ricardo de Sá Teles \\ Depto de Físico-Química, IQ, UNESP \\ Campus Araraquara \\ 14800-900, Araraquara, SP \\ E-mail: rteles@iq.unesp.br.
}

Resumo: Neste trabalho estudamos uma equação hiperbólica sem a hipótese de unicidade para o correspondente problema de Cauchy. Verificamos a existência de soluções fracas globais por meio do método das aproximações de Galerkin. E o nosso resultado principal é a construção do atrator de trajetórias para o problema considerado.

Palavras-chave: Atrator de trajetórias, solução fraca global, Faedo-Galerkin

Seja $\Omega$ um subconjunto aberto, limitado, conexo do $\mathbb{R}^{n}(n \geqslant 3)$ e com fronteira suave $\Gamma$. Para cada ponto $x \in \Gamma$, indicaremos por $\nu=\nu(x)$ o vetor normal exterior unitário a $\Gamma$ no ponto $x$. Consideremos o seguinte problema hiperbólico com condição de Neumann

$$
\left\{\begin{array}{l}
\frac{\partial^{2} u}{\partial t^{2}}+2 \gamma \frac{\partial u}{\partial t}=d \Delta u-f(u)+|u|^{\alpha-1} u \\
\frac{\partial u}{\partial \nu}=0 \quad(t, x) \in(0, \infty) \times \Gamma,
\end{array} \quad(t, x) \in(0, \infty) \times \Omega\right.
$$

onde $\gamma>0, d>0$ está fixado, $\alpha \in(0,1)$ e $f: \mathbb{R} \rightarrow \mathbb{R}$ é uma função de classe $C^{1}$ satisfazendo as seguintes hipóteses: existem constantes positivas $\gamma_{0}, \gamma_{1}, \gamma_{2}, c_{1}, c_{2}$ e $2<p<\frac{2 n}{n-2}$ tais que

$$
\begin{gathered}
|f(u)| \leqslant \gamma_{0}\left(|u|^{p-1}+1\right), \\
F(u)=\int_{0}^{u} f(w) d w, \quad F(u) \geqslant \gamma_{1}|u|^{p}-c_{1}, \\
f(u) \cdot u \geqslant \gamma_{2} F(u)-c_{2},
\end{gathered}
$$

para qualquer $u \in \mathbb{R}$.

Vamos especificar os espaços em que trabalharemos e definiremos quando uma função $u=$ $u(t, x)$ será solução de (1). Como não há um resultado de unicidade de solução para o problema (1) empregaremos a teoria do atrator de trajetórias em nosso estudo, adaptando resultados obtidos nas referências Chepyzhov, V. V. and Vishik, M. I. [1], [2], onde problemas similares a (1) foram considerados para a condição de Dirichlet. Os detalhes podem ser encontrados em R. S. Teles [3].

Consideremos $\tilde{f}(u)=f(u)-|u|^{\alpha-1} u$. Das hipóteses (2)-(4) podemos afirmar que existem constantes positivas $\tilde{\gamma_{0}}, \tilde{\gamma_{1}}, \tilde{\gamma_{2}}, \tilde{c_{1}}, \tilde{c_{2}}$ tais que

$$
|\tilde{f}(u)| \leqslant\left(\gamma_{0}+1\right)\left(|u|^{p-1}+1\right) \doteq \tilde{\gamma_{0}}\left(|u|^{p-1}+1\right) ;
$$




$$
\tilde{F}(u)=\int_{0}^{u} \tilde{f}(w) d w, \quad \tilde{F}(u) \geqslant \tilde{\gamma_{1}}|u|^{p}-\tilde{c_{1}},
$$

onde

$$
\begin{gathered}
\tilde{\gamma_{1}}=\frac{\gamma_{1}}{2} \quad \text { e } \quad \tilde{c_{1}}= \\
\tilde{c_{1}}+\frac{1}{\alpha+1}\left(\frac{2}{\gamma_{1} p}\right)^{\frac{\alpha+1}{p-(\alpha+1)}}\left(\frac{p-(\alpha+1)}{p}\right) ; \\
\tilde{f}(u) \cdot u \geqslant \tilde{\gamma_{2}}|u|^{p}-\tilde{c_{2}},
\end{gathered}
$$

onde

$$
\tilde{\gamma_{2}}=\frac{\gamma_{1} \gamma_{2}}{2} \quad \text { e } \quad \tilde{c_{2}}=\left[\gamma_{2} c_{1}+c_{2}+\left(\frac{2(\alpha+1)}{\gamma_{1} \gamma_{2} p}\right)^{\frac{\alpha+1}{p-(\alpha+1)}}\left(\frac{p-(\alpha+1)}{p}\right)\right]
$$

Faremos algumas observações antes de introduzir a definição de solução para o nosso problema.

Vamos considerar em $H^{1}(\Omega)$ a norma

$$
\|u\|^{2}=\int_{\Omega}\left(|u|^{2}+d|\nabla u|^{2}\right) d x
$$

para $u \in H^{1}(\Omega)$, que é equivalente a norma usual

$$
\|u\|_{H^{1}(\Omega)}^{2}=\|u\|_{L^{2}(\Omega)}^{2}+\|\nabla u\|_{L^{2}(\Omega)}^{2} .
$$

O $H^{1}(\Omega)$ munido da norma (8) será denotado por $H_{d}^{1}(\Omega)$.

O espaço dual de $H_{d}^{1}(\Omega)$ será denotado por $H_{d}^{-1}(\Omega)$ e consideraremos o operador contínuo

$$
\begin{aligned}
\Delta: H_{d}^{1}(\Omega) & \longrightarrow \\
u & \longmapsto\langle\varphi, \Delta u\rangle=-\int_{\Omega}^{-1} \nabla u \nabla \varphi d x
\end{aligned}
$$

Observemos que para $c_{3}=\tilde{c_{1}}+1$ existe uma constante positiva $K$ tal que a função $\Phi(u)=$ $\left(\tilde{F}(u)+c_{3}\right)^{\frac{1}{p}}$ é Lipschitz.

Seja $q$ o expoente conjugado de $p$, isto é, $\frac{1}{p}+\frac{1}{q}=1$. Observemos que se $u \in L^{\infty}\left(0, T ; L^{p}(\Omega)\right)$, então $|u|^{\alpha-1} u \in L^{\infty}\left(0, T ; L^{q}(\Omega)\right)$ e de (5) decorre que $\tilde{f}(u) \in L^{\infty}\left(0, T ; L^{q}(\Omega)\right)$. Além disso, existe uma constante $\tilde{\tilde{\gamma}}$ tal que

$$
\|\tilde{f}(u)\|_{L^{\infty}\left(0, T ; L^{q}(\Omega)\right)}^{q} \leqslant \tilde{\tilde{\gamma}}_{0}\left(\|u\|_{L^{\infty}\left(0, T ; L^{p}(\Omega)\right)}^{p}+1\right)
$$

para todo $T>0$, onde $\tilde{\gamma_{0}}=2^{q} \tilde{\gamma}_{0}^{q}(|\Omega|+1)$.

Por outro lado, se $u \in L^{\infty}\left(0, T ; H_{d}^{1}(\Omega)\right)$, então $\Delta u \in L^{\infty}\left(0, T ; H_{d}^{-1}(\Omega)\right)$. Como $p>2$, do Teorema de imersão de Sobolev e por dualidade temos $L^{q}(\Omega) \subset H^{-r}(\Omega)$, onde $r=\max \left\{1, n\left(\frac{1}{q}-\frac{1}{2}\right)\right\}$. Logo, se $u \in L^{\infty}\left(0, T ; L^{p}(\Omega)\right) \cap L^{\infty}\left(0, T ; H_{d}^{1}(\Omega)\right)$, temos que a equação (1) pode ser considerada no sentido de distribuição do espaço $D^{\prime}\left(\mathbb{R}_{+} ; H^{-r}(\Omega)\right)$.

Definição 1. Uma função $u=u(t, x), t \in[0, T], x \in \Omega$, é uma solução fraca global de (1) se $u \in L^{\infty}\left(0, T ; L^{p}(\Omega)\right) \cap L^{\infty}\left(0, T ; H_{d}^{1}(\Omega)\right), u_{t} \in L^{\infty}\left(0, T ; L^{2}(\Omega)\right)$ e u satisfaz a equação (1) no sentido de distribuição do espaço $D^{\prime}\left(0, T ; H^{-r}(\Omega)\right)$, para todo $T>0$, onde $r=\max \left\{1, n\left(\frac{1}{q}-\frac{1}{2}\right)\right\}$, isto é, para toda $\varphi \in H_{d}^{1}(\Omega) \cap L^{p}(\Omega)$ vale a seguinte igualdade

$$
\begin{aligned}
\frac{d^{2}}{d t^{2}} \int_{\Omega} u(t, x) \cdot \varphi(x) d x & +2 \gamma \frac{d}{d t} \int_{\Omega} u(t, x) \cdot \varphi d x+\int_{\Omega}[d \nabla u(t, x) \cdot \nabla \varphi(x)+f(u(t, x)) \cdot \varphi(x)- \\
& \left.-|u(t, x)|^{\alpha-1} u(t, x) \cdot \varphi(x)\right] d x=0 .
\end{aligned}
$$


Observação 1. Sabemos que se u é uma solução fraca global da equação (1), então $u \in L^{\infty}\left(0, T ; L^{p}(\Omega)\right) \cap L^{\infty}\left(0, T ; H_{d}^{1}(\Omega)\right) \hookrightarrow L^{\infty}\left(0, T ; L^{2}(\Omega)\right)$ e $u_{t} \in L^{\infty}\left(0, T ; L^{2}(\Omega)\right)$ para todo $T>0$, portanto $u \in C\left(0, T ; L^{2}(\Omega)\right)$. Temos também que $u_{t} \in L^{\infty}\left(0, T ; L^{2}(\Omega)\right) \hookrightarrow L^{\infty}\left(0, T ; H^{-r}(\Omega)\right)$ e $u_{t t} \in L^{\infty}\left(0, T ; H^{-r}(\Omega)\right)$ para todo $T>0$, donde concluímos que $u_{t} \in C\left(0, T ; H^{-r}(\Omega)\right)$.

Proposição 1. Se u é uma solução fraca global de (1), então

$$
u \in C_{w}\left(0, T ; H_{d}^{1}(\Omega)\right), \quad u \in C_{w}\left(0, T ; L^{p}(\Omega)\right), \quad u_{t} \in C_{w}\left(0, T ; L^{2}(\Omega),\right.
$$

para todo $T>0$. Além disso, para todo $\delta \in \mathbb{R}$ a função

$$
t \mapsto\|u(t)\|_{H_{d}^{1}(\Omega)}+\left\|u_{t}(t)+\delta u(t)\right\|_{L^{2}(\Omega)}+\|u(t)\|_{L^{p}(\Omega)}
$$

é semicontínua inferiormente para $t \in[0, T]$, para todo $T>0$.

Sejam $u \in H_{d}^{1}(\Omega) \cap L^{p}(\Omega)$ e $v \in L^{2}(\Omega)$. Definamos $I:\left(H_{d}^{1}(\Omega) \cap L^{p}(\Omega)\right) \times L^{2}(\Omega) \rightarrow \mathbb{R}$ por

$$
I(u, v)=\frac{1}{2} \int_{\Omega}\left[|v|^{2}+d|\nabla u|^{2}+2 \tilde{F}(u)\right] d x .
$$

Proposição 2. Existem constantes positivas $k_{1}, k_{2}, k_{3}, k_{4}$ tais que

$$
k_{1}\left[\|u\|_{H_{d}^{1}(\Omega)}^{2}+\|v\|_{L^{2}(\Omega)}^{2}+\|u\|_{L^{p}(\Omega)}^{p}\right]-k_{2} \leqslant I(u, v)
$$

$e$

$$
I(u, v) \leqslant k_{3}\left[\|u\|_{H_{d}^{1}(\Omega)}^{2}+\|v\|_{L^{2}(\Omega)}^{2}+\|u\|_{L^{p}(\Omega)}^{p}\right]+k_{4} .
$$

Demonstração. De fato, usando a hipótese (6) e a desigualdade de Young obtemos

$$
\begin{aligned}
I(u, v) & \geqslant \frac{1}{2} \min \left\{1, \tilde{\gamma_{1}}\right\}\left[\|u\|_{H_{d}^{1}(\Omega)}^{2}+\|v\|_{L^{2}(\Omega)}^{2}+\|u\|_{L^{p}(\Omega)}^{p}\right]- \\
& -\left[\tilde{c_{1}}|\Omega|+\frac{1}{2}\left(\frac{2}{\tilde{\gamma_{1}} p}\right)^{\frac{2}{p-2}}|\Omega|\left(\frac{p-2}{p}\right)\right] .
\end{aligned}
$$

Se $\tilde{L}$ é uma constante positiva tal que $|\tilde{F}(u)| \leqslant \tilde{L}\left(1+|u|^{p}\right)$, então

$$
I(u, v) \leqslant \frac{1}{2} \max \{1,2 \tilde{L}\}\left[\|u\|_{H_{d}^{1}(\Omega)}^{2}+\|v\|_{L^{2}(\Omega)}^{2}+\|u\|_{L^{p}(\Omega)}^{p}\right]+\tilde{L}|\Omega| .
$$

Portanto,

$$
\begin{gathered}
k_{1}=\frac{1}{2} \min \left\{1, \tilde{\gamma_{1}}\right\}, \quad k_{2}=\tilde{c_{1}}|\Omega|+\frac{1}{2}\left(\frac{2}{\tilde{\gamma_{1}} p}\right)^{\frac{2}{p-2}}|\Omega|\left(\frac{p-2}{p}\right) \\
k_{3}=\frac{1}{2} \max \{1,2 \tilde{L}\}, \quad k_{4}=\tilde{L}|\Omega| .
\end{gathered}
$$

Seja

$$
0<\delta<\min \left\{2 \gamma, 2 k_{1}\right\}
$$

onde $k_{1}=\frac{1}{2} \min \left\{1, \tilde{\gamma_{1}}\right\}$. Consideremos o seguinte funcional

$$
J_{\delta}(u, v)=\frac{1}{2} \int_{\Omega}\left[|v|^{2}+d|\nabla u|^{2}+2 \tilde{F}(u)+2 \delta u \cdot v+2 \delta \gamma|u|^{2}\right] d x .
$$


Proposição 3. O funcional $J_{\delta}$ satisfaz

$$
\left(k_{1}-\frac{\delta}{2}\right)\left[\|u\|_{H_{d}^{1}(\Omega)}^{2}+\|v\|_{L^{2}(\Omega)}^{2}+\|u\|_{L^{p}(\Omega)}^{p}\right]-k_{2} \leqslant J_{\delta}(u, v)
$$

$$
J_{\delta}(u, v) \leqslant\left(k_{3}+\frac{\delta}{2}+\delta \gamma\right)\left[\|u\|_{H_{d}^{1}(\Omega)}^{2}+\|v\|_{L^{2}(\Omega)}^{2}+\|u\|_{L^{p}(\Omega)}^{p}\right]+k_{4},
$$

onde as constantes $k_{1}, k_{2}, k_{3}, k_{4}$ foram definidas na Proposição 2.

Corolário 1. Se u é uma solução fraca global de (1), então a função real $z(t) \doteq J_{\delta}\left(u(t), u_{t}(t)\right)$ é semicontinua inferiormente no intervalo $[0, T]$, para todo $T>0$.

Teorema 1. Se u $:[0, \infty) \times \Omega \rightarrow \mathbb{R}$ é uma solução suficientemente regular de $(1)$ e $z(t)=$ $J_{\delta}\left(u(t), \partial_{t} u(t)\right)$, então existem constantes $\mu_{\delta}>0$ e $\rho_{\delta}>0$ tais que

$$
\frac{d}{d t} z(t)+\mu_{\delta} z(t) \leqslant \rho_{\delta}
$$

e portanto,

$$
z(t) \leqslant z(0) e^{-\mu_{\delta} t}+\frac{\rho_{\delta}}{\mu_{\delta}}
$$

Demonstração. Temos que

$$
\begin{aligned}
& \frac{d}{d t} J_{\delta}\left(u, u_{t}\right) \leqslant-2 \gamma \int_{\Omega}\left|u_{t}\right|^{2} d x+\delta \int_{\Omega}\left|u_{t}\right|^{2} d x-d \delta \int_{\Omega}|\nabla u|^{2} d x-\tilde{\gamma_{2}} \delta \int_{\Omega}|u|^{p} d x+\delta \tilde{c_{2}}|\Omega|+ \\
& \leqslant-\min \left\{2 \gamma-\delta, \delta, \frac{\delta \tilde{\gamma_{2}}}{2}\right\}\left[\left\|u_{t}\right\|_{L^{2}(\Omega)}^{2}+\|u\|_{H_{d}^{1}(\Omega)}^{2}+\|u\|_{L^{p}(\Omega)}^{p}\right]+\delta\left[\tilde{c_{2}}|\Omega|+\left(\frac{4}{\tilde{\gamma_{2}} p}\right)^{\frac{2}{p-2}}|\Omega| \frac{p-2}{p}\right] .
\end{aligned}
$$

De $(20)$ e $(17)$, obtemos

$$
\frac{d}{d t} J_{\delta}\left(u, u_{t}\right) \leqslant-k_{5} J_{\delta}\left(u, u_{t}\right)+k_{6},
$$

onde

$$
k_{5}=\frac{\min \left\{2 \gamma-\delta, \delta, \delta \tilde{\gamma_{2}}\right\}}{k_{3}+\frac{\delta}{2}+\delta \gamma}, \quad k_{6}=k_{4} k_{5}+\delta\left[\tilde{c_{2}}|\Omega|+\left(\frac{4}{\tilde{\gamma_{2}} p}\right)^{\frac{2}{p-2}}|\Omega| \frac{p-2}{p}\right]
$$

e as constantes $k_{3}$ e $k_{4}$ foram dadas na Proposição 2 .

E de (21) concluímos que

$$
z(t) \leqslant z(0) e^{-k_{5} t}+\frac{k_{6}}{k_{5}}
$$

ou seja, $\mu_{\delta}=k_{5}$ e $\rho_{\delta}=k_{6}$.

Fixemos $N>0$ e vamos definir o espaço de trajetórias $\mathcal{K}_{d}^{+}(N)$ da equação (1).

Definição 2. $O$ espaço $\mathcal{K}_{d}^{+}(N)$ é o conjunto das soluções fracas globais u da equação (1) que verificam a desigualdade

$$
z(t) \leqslant N e^{-\mu_{\delta} t}+\frac{\rho_{\delta}}{\mu_{\delta}},
$$

para todo $t \geqslant 0$, onde $z(t)=J_{\delta}\left(u(t), \partial_{t} u(t)\right)$ e $\delta$ foi dado em (14). 
Proposição 4. Se $u_{0} \in H_{d}^{1}(\Omega) \cap L^{p}(\Omega), u_{1} \in L^{2}(\Omega)$ e $z_{0}=J_{\delta}\left(u_{0}, u_{1}\right) \leqslant N$, então existe pelo menos uma solução fraca global $u \in \mathcal{K}_{d}^{+}(N)$ tal que

$$
\left.u\right|_{t=0}=u_{0},\left.\quad \partial_{t} u\right|_{t=0}=u_{1} .
$$

Vamos definir os conjuntos $\mathcal{F}_{l o c, d}^{+}, \mathcal{F}_{b, d}^{+}$e a topologia $\Theta_{l o c, d}^{+}$associados à equação (1). Começamos definindo o conjunto $\mathcal{F}_{T, d}$ da seguinte forma

$$
\begin{aligned}
\mathcal{F}_{T, d}= & \left\{v: v \in L^{\infty}\left(0, T ; L^{p}(\Omega)\right) \cap L^{\infty}\left(0, T ; H_{d}^{1}(\Omega)\right), \partial_{t} v \in L^{\infty}\left(0, T ; L^{2}(\Omega)\right),\right. \\
& \left.\partial_{t}^{2} v \in L^{\infty}\left(0, T ; H^{-r}(\Omega)\right)\right\}
\end{aligned}
$$

e o munimos da topologia $\Theta_{T, d}$ que, em termos de sequências, é descrita da seguinte maneira: uma sequência $\left\{v_{m}\right\} \subset \mathcal{F}_{T, d}$ converge para uma função $v \in \mathcal{F}_{T, d}$ em $\Theta_{T, d}$ se $v_{m} \stackrel{*}{\rightarrow} v$ em $L^{\infty}\left(0, T ; H_{d}^{1}(\Omega)\right) \cap L^{\infty}\left(0, T ; L^{p}(\Omega)\right), \partial_{t} v_{m} \stackrel{*}{\rightarrow} \partial_{t} v$ em $L^{\infty}\left(0, T ; L^{2}(\Omega)\right)$ e $\partial_{t}^{2} v_{m} \stackrel{*}{\rightarrow} \partial_{t}^{2} v$ em $L^{\infty}\left(0, T ; H^{-r}(\Omega)\right)$.

A topologia definida acima torna $\mathcal{F}_{T, d}$ um espaço topológico de Hausdorff e Frechet-Uryshon com base topológica enumerável.

O conjunto $\mathcal{F}_{T, d}$ e a topologia $\Theta_{T, d}$ dão origem aos conjuntos $\mathcal{F}_{l o c, d}^{+}, \mathcal{F}_{b, d}^{+}$e a topologia $\Theta_{l o c, d}^{+}$. Temos que

$$
\begin{aligned}
& \mathcal{F}_{l o c, d}^{+}=\left\{v: v \in L_{l o c}^{\infty}\left(\mathbb{R}_{+} ; L^{p}(\Omega)\right) \cap L_{l o c}^{\infty}\left(\mathbb{R}_{+} ; H_{d}^{1}(\Omega)\right), \partial_{t} v \in L_{l o c}^{\infty}\left(\mathbb{R}_{+} ; L^{2}(\Omega)\right) \mathrm{e}\right. \\
&\left.\partial_{t}^{2} v \in L_{l o c}^{\infty}\left(\mathbb{R}_{+} ; H^{-r}(\Omega)\right)\right\} ; \\
& \mathcal{F}_{b, d}^{+}=\left\{v \in \mathcal{F}_{l o c, d}^{+}: v \in L^{\infty}\left(\mathbb{R}_{+} ; L^{p}(\Omega)\right) \cap L^{\infty}\left(\mathbb{R}_{+} ; H_{d}^{1}(\Omega)\right) \cap \partial_{t} v \in L^{\infty}\left(\mathbb{R}_{+} ; L^{2}(\Omega)\right) \mathrm{e}\right. \\
&\left.\partial_{t}^{2} v \in L^{\infty}\left(\mathbb{R}_{+} ; H^{-r}(\Omega)\right) \operatorname{com}\|v\|_{\mathcal{F}_{b, d}^{+}}<+\infty\right\},
\end{aligned}
$$

onde

$$
\|v\|_{\mathcal{F}_{b, d}^{+}}=\|v\|_{L^{\infty}\left(\mathbb{R}_{+} ; L^{p}(\Omega)\right)}+\|v\|_{L^{\infty}\left(\mathbb{R}_{+} ; H_{d}^{1}(\Omega)\right)}+\left\|\partial_{t} v\right\|_{L^{\infty}\left(\mathbb{R}_{+} ; L^{2}(\Omega)\right)}+\left\|\partial_{t}^{2} v\right\|_{L^{\infty}\left(\mathbb{R}_{+} ; H^{-r}(\Omega)\right)}
$$

E a topologia $\Theta_{l o c, d}^{+}$em $\mathcal{F}_{l o c, d}^{+}$é definida da seguinte forma: $v_{m} \rightarrow v$ em $\Theta_{l o c, d}^{+}$quando $\Pi_{[0, T]} v_{m} \rightarrow$ $\Pi_{[0, T]} v$ em $\Theta_{T, d}$ para todo $T>0$.

O espaço $\mathcal{F}_{l o c, d}^{+}$munido da topologia $\Theta_{l o c, d}^{+}$é um espaço topológico Hausdorff, FrechetUryshon e possui uma base enumerável.

O espaço $\mathcal{K}_{d}^{+}(N)$ munido da topologia $\Theta_{l o c, d}^{+}$é o espaço de trajetórias da equação (1).

Definamos a aplicação $T(t): \mathcal{F}_{l o c, d}^{+} \rightarrow \mathcal{F}_{l o c, d}^{+}$por $T(t) u(s)=u(t+s)$ e consideremos a família $\{T(t): t \geqslant 0\}$ que constitui um semigrupo. Como a equação (1) é autônoma temos que $\mathcal{K}_{d}^{+}(N)$ é invariante pelo semigrupo $\{T(t): t \geqslant 0\}$, logo podemos considerar $T(t): \mathcal{K}_{d}^{+}(N) \rightarrow \mathcal{K}_{d}^{+}(N)$.

Proposição 5. Se $\mathcal{K}_{d}^{+}(N)$ é o espaço de trajetórias da equação (1), então para toda função $u \in \mathcal{K}_{d}^{+}(N)$ temos que $T(t) u \in \mathcal{K}_{d}^{+}(N)$ para $t \geqslant 0$.

Proposição 6. Se $\mathcal{K}_{d}^{+}(N)$ é o espaço de trajetórias da equação $(1)$, então $\mathcal{K}_{d}^{+}(N) \subset \mathcal{F}_{b, d}^{+}$.

Proposição 7. O espaço de trajetórias $\mathcal{K}_{d}^{+}(N)$ é fechado na topologia $\Theta_{\text {loc,d }}^{+}$.

Agora, fixemos um valor $\delta=\delta_{0}$ verificando (14). Vamos definir o conjunto

$$
P=\left\{u \in \mathcal{F}_{b, d}^{+}: z(t)=J_{\delta_{0}}\left(u, \partial_{t} u\right) \leqslant 2 \frac{\rho_{\delta_{0}}}{\mu_{\delta_{0}}}\right\} .
$$

Observemos que o conjunto $P$ é tal que $\|u\|_{\mathcal{F}_{b, d}^{+}}<+\infty$ para toda função $u \in \mathcal{F}_{b, d}^{+}$e é compacto em $\Theta_{l o c, d}^{+}$. Além disso, a desigualdade (19) implica que o conjunto $P$ é um conjunto absorvente do espaço $\mathcal{K}_{d}^{+}(N)$. 
Proposição 8. Se $\tilde{f}$ satisfaz (5)-(7), então para cada d $>0$ a equação (1) possui um atrator de trajetórias $\mathfrak{A}_{d}(N)$.

Definamos os conjuntos

$$
\begin{aligned}
\mathcal{F}_{l o c, d}= & \left\{v: v \in L_{l o c}^{\infty}\left(\mathbb{R} ; L^{p}(\Omega)\right) \cap L_{l o c}^{\infty}\left(\mathbb{R} ; H_{d}^{1}(\Omega)\right), \partial_{t} v \in L_{l o c}^{\infty}\left(\mathbb{R} ; L^{2}(\Omega)\right) \mathrm{e}\right. \\
& \left.\partial_{t}^{2} v \in L_{l o c}^{\infty}\left(\mathbb{R} ; H^{-r}(\Omega)\right)\right\} ; \\
\mathcal{F}_{b, d}= & \left\{v: v \in L^{\infty}\left(\mathbb{R} ; L^{p}(\Omega)\right) \cap L^{\infty}\left(\mathbb{R} ; H_{d}^{1}(\Omega)\right) \cap \partial_{t} v \in L^{\infty}\left(\mathbb{R} ; L^{2}(\Omega)\right) \mathrm{e}\right. \\
& \left.\partial_{t}^{2} v \in L^{\infty}\left(\mathbb{R} ; H^{-r}(\Omega)\right) \operatorname{com}\|v\|_{\mathcal{F}_{b, d}}<+\infty\right\},
\end{aligned}
$$

onde

$$
\|v\|_{\mathcal{F}_{b, d}}=\|v\|_{L^{\infty}\left(\mathbb{R} ; L^{p}(\Omega)\right)}+\|v\|_{L^{\infty}\left(\mathbb{R} ; H_{d}^{1}(\Omega)\right)}+\left\|\partial_{t} v\right\|_{L^{\infty}\left(\mathbb{R} ; L^{2}(\Omega)\right)}+\left\|\partial_{t}^{2} v\right\|_{L^{\infty}\left(\mathbb{R} ; H^{-r}(\Omega)\right)} ;
$$

E munimos $\mathcal{F}_{l o c, d}$ com a topologia $\Theta_{l o c, d}$ definida da seguinte forma: $v_{m} \rightarrow v$ em $\Theta_{l o c, d}$ quando $\Pi_{[-T, T]} v_{m} \rightarrow \Pi_{[-T, T]} v$ em $\Theta_{[-T, T], d}$ para todo $T>0$.

Denotamos por $\mathcal{K}_{d}$ o núcleo da equação (1) consistindo de todas as soluções fracas $u$ da equação

$$
\partial_{t}^{2} u+2 \gamma \partial_{t} u=d \Delta u-f(u)+|u|^{\alpha-1} u, \quad t \in \mathbb{R},
$$

limitadas na norma do espaço $\mathcal{F}_{b, d}$ e satisfazendo a desigualdade

$$
z(t) \leqslant J_{\delta}\left(u(t), u_{t}(t)\right) \leqslant \frac{\rho_{\delta}}{\mu_{\delta}},
$$

para todo $t \in \mathbb{R}$.

Teorema 2. Seja $\mathcal{K}_{d}^{+}(N)$ o espaço de trajetórias da equação (1) para $N>0$ fixado. Se $\mathfrak{A}_{d}(N) \subset$ $P$ é o atrator de trajetórias do semigrupo translação $T(t): \mathcal{K}_{d}^{+}(N) \rightarrow \mathcal{K}_{d}^{+}(N)$, então o conjunto $\mathfrak{A}_{d}(N)$ é limitado na norma do espaço $\mathcal{F}_{b, d}^{+}$e é compacto em relação a topologia $\Theta_{\text {loc,d }}^{+}$. Além disso,

$$
\mathfrak{A}_{d}=\Pi_{+} \mathcal{K}_{d}
$$

$O$ conjunto $\mathcal{K}_{d}$ é limitado em $\mathcal{F}_{b, d}$ e compacto em $\Theta_{\text {loc,d }}$.

Proposição 9. o atrator de trajetórias $\mathfrak{A}_{d}(N)$ não depende de $N>0$, isto é, $\mathfrak{A}_{d}(N) \equiv \mathfrak{A}_{d}$.

\section{Referências}

[1] V. V. Chepyzhov \& M. I. Vishik, Attractors for equations of mathematical physics, Colloquium publications, 49, American Mathematical Society, Providence, RI, 2002.

[2] V. V. Chepyzhov \& M. I. Vishik, Global attractor and its perturbations for a dissipative hyperbolic equation, Russian Journal of Mathematical Physics, 8, (2001) 311-330.

[3] R. S. Teles, Atratores de trajetórias para algumas classes de equações diferenciais parciais, Tese de Doutorado, IME-USP, 2012. 